\title{
Hyperbaric oxygen therapy for spinal cord ischaemia after complex aortic repair - a retrospective review
}

\author{
Matteo Parotto ${ }^{1}$, Maral Ouzouniann ${ }^{2}$, Ludwik Fedorko ${ }^{1}$, George Oreopoulos ${ }^{3}$, Thomas Lindsay ${ }^{3}$, \\ Rita Katznelson ${ }^{4}$ \\ ${ }^{1}$ Department of Anaesthesia and Pain Management, Toronto General Hospital/University Health Network, \\ Toronto, Ontario, Canada \\ ${ }^{2}$ Division of Cardiac Surgery, Peter Munk Cardiac Centre, University Health Network and Department \\ of Surgery, University of Toronto, Toronto, Ontario, Canada \\ ${ }^{3}$ Division of Vascular Surgery, Toronto General Hospital, Toronto, Ontario, Canada \\ ${ }^{4}$ Hyperbaric Medicine Unit, Department of Anaesthesia and Pain Management, Toronto General Hospital, \\ Toronto, Ontario, Canada
}

\begin{abstract}
Background: Complex aortic repair (CAR) carries high rates of debilitating postoperative complications, including spinal cord injury. The rate of spinal cord deficits post-CAR is approximately $10 \%$, with permanent paraplegia in $2.9 \%$ and paraparesis in $2.4 \%$ of patients. Treatment options are limited. Rescue therapies include optimization of spinal cord perfusion and oxygen delivery by mean arterial pressure augmentation ( $>90 \mathrm{~mm} \mathrm{Hg}$ ), cerebrospinal fluid drainage, and preservation of adequate haemoglobin concentration $\left(>100 \mathrm{~g} \mathrm{~L}^{-1}\right)$. Hyperbaric oxygen therapy (HBOT) has been described in several case reports as part of the multimodal treatment for spinal cord ischemia.

HBOT has been used in our centre as adjunct rescue treatment for patients with spinal cord injury post-CAR that were refractory to traditional medical management, and we aimed to retrospectively review these cases.

Methods: After Research Ethics Board approval, we performed a retrospective review of all post-CAR patients who developed spinal cord injury with severe motor deficit and were treated with HBOT at our institution since 2013.

Results: Seven patients with spinal cord injury after CAR were treated with HBOT in addition to traditional rescue therapies. Five patients showed varying degrees of recovery, with two displaying full recovery. One developed oxygeninduced seizure, medically treated. No other HBOT-related complications were noted.

Conclusions: Our retrospective study shows a potential benefit of hyperbaric oxygen therapy on neurological outcome in patients who developed spinal cord injury after CAR. Prospective research is needed to understand the role, efficacy, benefits and risks of HBOT in this setting.
\end{abstract}

Anaesthesiology Intensive Therapy 2018, vol. 50, no 2, 103-109

Key words: spinal cord ischaemia, hyperbaric oxygen therapy, aortic aneurysm

Distal Arch and Thoracoabdominal aortic aneurysms (TAAA) represent an extremely challenging clinical entity [1]. Affected patients risk life-threatening rupture without surgical intervention. Complex aortic repair (CAR) for this pathology carries substantial risk, including a risk of death or, in those who survive, life-altering complications such as stroke, paraplegia, and renal failure necessitating dialysis [2].
A recently published retrospective data collection of a large cohort of patients who underwent CAR for TAAA at a single institution reported a rate of spinal cord deficits of $9.6 \%$, with permanent paraplegia in $2.9 \%$, and permanent paraparesis in $2.4 \%$ of the patients [2]. This could appear as an immediate intraoperative or perioperative event or as a delayed finding, materialising up to several days after surgery. Permanent neurological deficit is a major cause 
of morbidity and leads to decreased long-term survival of patients with CAR [3, 4].

The causes leading to spinal cord injury in aortic repairs include infarction of the spinal cord from direct interruption of the cord blood supply or a critical collateral, systemic hypoperfusion, atheromatous embolic infarction, or spontaneous thrombosis of an atherosclerotic radicular artery, and ischemia-reperfusion injury that progresses to infarction [4-7].

The rescue therapies in spinal cord injury post CAR include optimization of spinal cord perfusion and oxygen delivery by the following: mean arterial pressure (MAP) augmentation (typically $>90 \mathrm{~mm} \mathrm{Hg}$ ), cerebrospinal fluid (CSF) pressure decrease by spinal cerebrospinal fluid (CSF) drainage and preservation of adequate haemoglobin concentration (typically $>100 \mathrm{~g} \mathrm{~L}^{-1}$ ) [6].

Hyperbaric oxygen therapy (HBOT) is a medical treatment based on an intermittent inhalation of $100 \%$ oxygen in a hyperbaric chamber at a pressure higher than one absolute atmosphere (ATA, 1 ATA $=760 \mathrm{~mm} \mathrm{Hg}$, normal atmospheric pressure at sea level). It is currently used in clinical practice in a number of urgent and elective indications. HBOT is a safe, non-invasive intervention, and the only absolute contraindication is a non-treated pneumothorax. Physiological effects of HBOT are based on a marked increase in the amount of dissolved oxygen carried by the blood. High plasma oxygen concentration enhances the rate and distance of oxygen diffusion into ischaemic areas with compromised circulation. Long-lasting consequences of HBOT are related to the stimulating effect of supra-physiological oxygen concentrations on a variety of biochemical processes. HBOT activates oxidant-antioxidant mechanisms with nitric oxide playing a key role [8] and such stimulates secretion of growth factors, vascular endothelia growth factor, hypoxia-inducible factor-1, and stem cells. By activating signal transduction cascades HBOT modifies inflammatory response and improves ischaemic-reperfusion injury [9].

Therefore, HBOT has the potential for several positive effects in spinal cord deficits after CAR, such as correction of tissue hypoxia, modulation of ischaemia-reperfusion injury and reduction of inflammatory oedema. Case reports have previously described HBOT use in this setting [10].

We report a retrospective review of a single centre experience with the use of HBOT as an adjuvant treatment for acute spinal cord deficit after CAR.

\section{METHODS}

Institutional Research Ethics Board approval was obtained. The requirement for written informed consent was waived by the ethics committee, given the retrospective analysis nature of our study. We performed a retrospective review of the clinical details and outcomes of all post-CAR patients that developed spinal cord injury with severe mo- tor deficit and were treated with $\mathrm{HBOT}$ at our institution since 2013. HBOT was applied at 2.4-2.8 ATA (Table 1) once or twice daily until complete neurological recovery or symptoms plateau. The patients were treated in a multiplace hyperbaric chamber (Fink engineering PTY LTD, rectangular Hyperbaric System, Australia) that can accommodate two critically ill intubated ventilated patients at once. The chamber is equipped with a ventilator (IPER Hyperbaric Ventilator, Siaretron 1000, Italy), monitor (CARESCAPE B650, GE Medical Systems, Worldwide) and infusion pumps (CAREFUSION Alaris PC infusion pumps, Guardrails, BD, Worldwide) that are compatible with a hyperbaric environment. During hyperbaric oxygen therapy, patients were managed by two attendants inside the chamber, namely a respiratory therapist and hyperbaric technologist. The management of patient sedation (if needed), haemodynamic parameters and CSF drainage was not different from intensive care unit management. Two additional hyperbaric technologists, namely a controller and an assistant controller, operated the chamber outside. A hyperbaric physician, trained in cardiac anaesthesia and intensive care medicine, and an intensive care nurse, were present in the unit supervising the patient from outside of the chamber. The hyperbaric physician was ready to enter the chamber if necessary.

Patient characteristics including age, gender and comorbidities were recorded. Baseline and postoperative labs were also recorded, along with pertinent surgical data and postoperative data that include duration of cardiopulmonary bypass, blood transfusions, haemodynamic and respiratory data, timing of spinal cord deficits and rescue therapies, HBOT treatment and outcome.

\section{RESULTS}

A total of seven patients ( 6 males, 1 female) with spinal cord injury after CAR were treated with HBOT when traditional rescue therapies (MAP augmentation, $\mathrm{Hb}>100 \mathrm{~g} \mathrm{~L}^{-1}$ and/or CSF drainage) had failed. A summary of relevant data and treatment details is described in Table 1. The onset of spinal cord injury varied from intraoperative to 7 days postoperatively. The time from the onset of spinal cord injury signs/symptoms to the institution of HBOT ranged from 8 hours to 30 hours.

Two patients died from perioperative complications (1 developed multiple organ failure related to sepsis, $1 \mathrm{had}$ an extensive myocardial infarction that led to cardiac arrest). Five patients showed varying degrees of neurological recovery, with two patients displaying complete recovery. One patient developed oxygen induced seizures (1 episode of 1 minute duration) while receiving HBOT treatment; the seizures resolved after intravenous midazolam ( $2 \mathrm{mg}$ ) administration. No other HBOT-related complications were noted.

A brief description of each individual case is provided in supplemental results sections. 


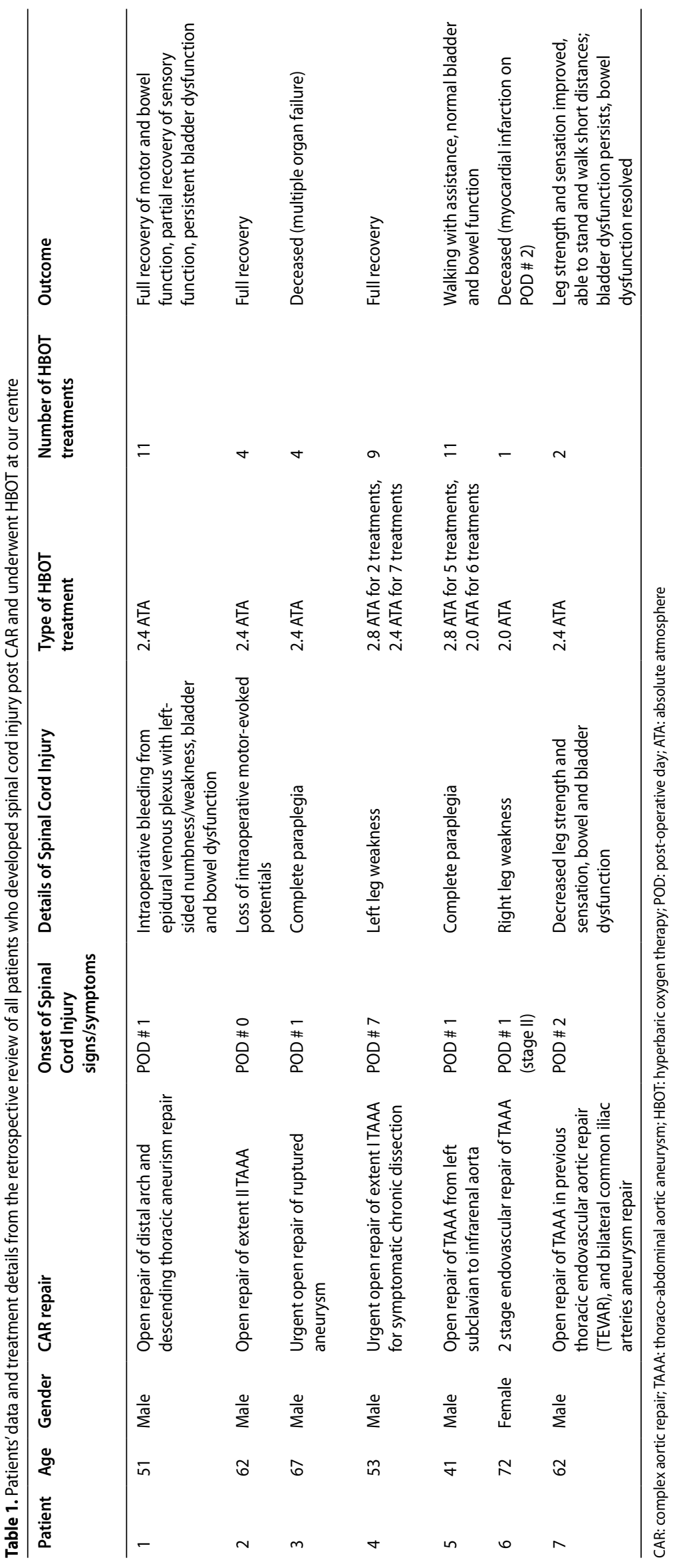




\section{DISCUSSION}

We present a retrospective case series describing the beneficial effect of HBOT in spinal cord injury after CAR. To our knowledge, this is the first series described in the literature. The patients included in this retrospective review presented different degrees of spinal cord injury and showed improvement in neurological function after HBOT, when traditional rescue therapies (MAP augmentation, $\mathrm{Hb}$ $>100 \mathrm{~g} \mathrm{~L}^{-1}$ and/or CSF drainage) had failed. No major HBOT complications were observed, with 1 patient experiencing a single seizure episode that promptly responded to medical management.

It is important to notice that our retrospective case series has several limitations, including the relatively low number of patients, the heterogeneity of HBOT (frequency, duration, ATA) utilized and the variable outcomes observed.

However, a growing body of evidence is emerging indicating that hyperbaric oxygen therapy, alone or in combination with other therapeutic modalities, may have positive effects in different types of spinal cord injury.

Wajima et al. [11] reported successful treatment of acute epidural haematoma at L3-L5 level after epidural steroid injection. The patient was treated with HBOT immediately after developing paraplegia and experienced complete neurological recovery without surgical intervention after 5 daily HBOTs. Kohshi et al. [12] described the conservative treatment of spinal epidural abscess causing spinal cord compression at the $\mathrm{C} 1-\mathrm{C} 4$ level. The addition of HBOT to the antibiotic therapy caused a dramatic improvement in the neurological symptoms with complete neurological recovery. Combination of HBOT, thrombolysis and hypothermia prevented permanent neurological injury and paralysis in an oncological patient with acute spinal cord ischaemia due to vertebral artery occlusion [13].

Several case reports have demonstrated the therapeutic effect of HBOT in patients with early and delayed spinal cord ischaemia after CAR [10, 14, 15]. Puttaswami et al. [10] described complete resolution of postoperative paraplegia after treatment with HBOT in a patient who underwent resection of type II thoraco-abdominal aneurysm. Another case report of paraplegia after emergent repair of type A aortic dissection showed that HBOT led to significant neurological improvement after a lack of response for 3 days of combined management with cerebrospinal fluid drainage, steroids and mannitol [14]. Morishito et al. [15] described urgent HBOT in a case of delayed paraplegia that developed 15 days after axillo-bifemoral bypass for acute Type B aortic dissection. The patient recovered with no residual neurological compromise after being treated with hyperbaric oxygen for 2 days.

The mechanisms of the beneficial effects of HBOT in spinal cord injury after CAR have been studied in animal models of spinal cord injury. It is postulated that by increasing oxygen delivery to the ischaemic spinal cord, HBOT reduces hypoxia and hypoxia-induced apoptosis, corrects acidosis and improves microcirculation, limits axonal death and promotes axonal regeneration. All these effects result in better clinical recovery of compromised neurological function. The time and number of administered HBOTs, as well as its combination with other therapeutic interventions, could significantly affect post injury outcomes.

Animal studies have revealed that early HBOT administration attenuates the death of motor neurons in rabbits with spinal cord ischaemia. Single HBOT applied 30 minutes after the ischaemic insult has a better neuro-protective effect than HBOT administered 3 hours post injury. However, repeated HBOTs can significantly expand the therapeutic window for HBOT application. In a rat model of spinal cord injury, animals treated with serial HBOTs started 6 hours post injury showed better neurological recovery in comparison to animals who received a single HBOT early (30 min or 3 hours post-injury). The authors concluded that serial HBOTs have beneficial effects, even hours after the injury, and are superior to single hyperbaric oxygen exposure. This observation was confirmed by another report where rats with acute spinal cord lesion were treated 30 minutes or 24 hours after injury for 10 days with hourly HBOTs at 2.0 ATA. Both groups demonstrated better functional recovery in comparison to control (non-treated) group. There was no clinical difference in neurological examination at one and two weeks postinjury between the group that had started HBOTs 30 minutes after the insult and the group that started treatments with a 24 hour delay. The combined administration of HBOT with hypothermia caused higher activity of antioxidant enzymes in the injured spinal cord tissue than HBOT or hypothermia alone. It was also more effective than methylprednisolone treatment [16]. The addition of HBOT to stem cell transplantation had a synergistic effect on axon regeneration and functional recovery in rats with spinal cord injury [17].

The molecular mechanisms of HBOT in spinal cord injury include effects on apoptosis, hypoxia-inducible factor (HIF)-1alpha and neurotrophic growth factors, as well as modification of local inflammatory response in the injured tissue. Rats with compression spinal cord injury treated with HBOT immediately after the insult had smaller spinal cord infarction, less apoptosis, decreased spinal cord production of interleukin-1 beta and tumour necrosis factor-alpha, lower expression of HIF-1alpha and higher production of interleukin-10 and vasculo-endothelial and glial cell line-derived neurotrophic growth factors in comparison with untreated controls [18-20]. These findings correlated with the better motor function scores in the HBOT treated animals $[18,19]$. The iNOS mRNA-iNOS-NO signalling pathway could also be involved in HBOT induced neuro-protection [21]. 
The anti-inflammatory effect of HBOT in spinal cord injury has been observed in numerous studies. This may be related to macrophage polarization and their ability to secrete proand anti-inflammatory cytokines [22]. It also could be result of the down-regulation of several signalling pathways such as TLR2/NF-KB and HMGB1/NF-kB that are accountable for the activation of inflammatory response [23, 24].

HBOT reduces the expression of neuronal genes and proteins responsible for local oedema in areas of spinal cord injury. It improves local microenvironment, stimulates growth of unmyelinated and myelinated nerve fibres and such promotes clinical neurological recovery [25].

\section{CONCLUSIONS}

In summary, HBOT has multiple molecular and biochemical effects in the injured spinal cord. In the retrospective review of patients who developed spinal cord injury after CAR in our institution, we observed improved neurological outcomes with HBOT treatment, after the failure of traditional rescue techniques.

Larger retrospective data reviews and randomized controlled trials are urgently needed to clarify the role of this non-invasive, safe therapeutic modality in the management of ischaemic spinal cord lesions after significant vascular interventions.

\section{ACKNOWLEDGEMENTS}

1. Source of funding: departmental resources.

2. Conflict of interest: none.

\section{References:}

1. Juvonen T, Ergin MA, Galla JD, et al. Prospective study of the natural history of thoracic aortic aneurysms. Ann Thorac Surg. 1997; 63(6): 1533-1545, indexed in Pubmed: 9205145.

2. Coselli JS, LeMaire SA, Preventza O, et al. Outcomes of 3309 thoracoabdominal aortic aneurysm repairs. J Thorac Cardiovasc Surg. 2016; 151(5): 1323-1337, doi: 10.1016/j.jtcvs.2015.12.050, indexed in Pubmed: 26898979

3. Crawford ES, Svensson LG, Hess KR, et al. A prospective randomized study of cerebrospinal fluid drainage to prevent paraplegia after high-risk surgery on the thoracoabdominal aorta. J Vasc Surg. 1991; 13(1): 36-45; discussion 45, indexed in Pubmed: 1987395.

4. Svensson LG, Crawford ES, Hess KR, et al. Experience with 1509 patients undergoing thoracoabdominal aortic operations. J Vasc Surg. 1993; 17(2): 357-68; discussion 368, indexed in Pubmed: 8433431.

5. Chaikof EL, Salam AA. Neurologic complications of abdominal aortic reconstruction. In: Ernst CB, Stanley JC, editors. Current therapy in vascular surgery. 3rd ed. St Louis: Mosby-Year Book, Inc. ; 1995: 278-82.

6. Wynn MM, Acher CW. A modern theory of spinal cord ischemia/injury in thoracoabdominal aortic surgery and its implications for prevention of paralysis. J Cardiothorac Vasc Anesth. 2014; 28(4): 1088-1099, doi: 10.1053/j.jvca.2013.12.015, indexed in Pubmed: 25107722.

7. Rosenthal D. Spinal cord ischemia after abdominal aortic operation: is it preventable? J Vasc Surg. 1999; 30(3): 391-397, indexed in Pubmed: 10477631.

8. Venetsanou K, Fildissis G, Tokta R, et al. The role of nitric oxide in cellular response to hyperbaric conditions. Eur J Appl Physiol. 2012; 112(2):677687, doi: 10.1007/s00421-011-2027-8, indexed in Pubmed: 21656226.

9. Al-Waili NS, Butler GJ. Effects of hyperbaric oxygen on inflammatory response to wound and trauma: possible mechanism of action. ScientificWorldJournal. 2006; 6: 425-441, doi: 10.1100/tsw.2006.78, indexed in Pubmed: 16604253.

10. Puttaswamy V, Bennett M, Frawley JE. Hyperbaric oxygenation treatment of acute paraplegia after resection of a thoracoabdominal aortic aneurysm. J Vasc Surg. 1999; 30(6): 1158-1161, indexed in Pubmed: 10587403.

11. Wajima Z, Aida S. Does hyperbaric oxygen have positive effect on neurological recovery in spinal-epidural haematoma? A case report. $\mathrm{Br}$ J Anaesth. 2011; 107(6): 1006-1008, doi: 10.1093/bja/aer376, indexed in Pubmed: 22088875.

12. Kohshi K, Abe H, Mizoguchi Y, et al. Successful treatment of cervical spinal epidural abscess by combined hyperbaric oxygenation. Mt Sinai J Med. 2005; 72(6): 381-384, indexed in Pubmed: 16358162.

13. Lee $K$, Strozyk D, Rahman $C$, et al. Acute spinal cord ischemia: treatment with intravenous and intra-arterial thrombolysis, hyperbaric oxygen and hypothermia. Cerebrovasc Dis. 2010; 29(1): 95-98, doi: 10.1159/000259618, indexed in Pubmed: 19923816.

14. Yamashiro S, Kuniyoshi Y, Miyagi K, et al. Acute postoperative paraplegia complicating with emergency graft replacement of the ascending aorta for the type a dissection. Ann Thorac Cardiovasc Surg. 2003; 9(5): 330-333, indexed in Pubmed: 14672532.

15. Morishita A, Tomioka H, Katahira S, et al. Delayed visceral and spinal cord malperfusion after axillo-bifemoral bypass for complicated acute type B aortic dissection. Ann Vasc Dis. 2014; 7(3): 331-334, doi: 10.3400/ avd.cr.14-00059, indexed in Pubmed: 25298840.

16. Topuz K, Colak A, Cemil B, et al. Combined hyperbaric oxygen and hypothermia treatment on oxidative stress parameters after spinal cord injury: an experimental study. Arch Med Res. 2010; 41(7): 506-512, doi: 10.1016/j.arcmed.2010.10.004, indexed in Pubmed: 21167389.

17. Geng $\mathrm{CK}, \mathrm{Cao} \mathrm{HH}$, Ying $\mathrm{X}$, et al. Effect of mesenchymal stem cells transplantation combining with hyperbaric oxygen therapy on rehabilitation of rat spinal cord injury. Asian Pac J Trop Med. 2015; 8(6): 468-473, doi: 10.1016/j.apjtm.2015.05.001, indexed in Pubmed: 26194832.

18. Tai PA, Chang CK, Niu KC, et al. Attenuating experimental spinal cord injury by hyperbaric oxygen: stimulating production of vasculoendothelial and glial cell line-derived neurotrophic growth factors and interleukin-10. J Neurotrauma. 2010; 27(6): 1121-1127, doi: 10.1089/ neu.2009.1162, indexed in Pubmed: 20334467.

19. Zhou Yi, Liu Xh, Qu Sd, et al. Hyperbaric oxygen intervention on expression of hypoxia-inducible factor-1a and vascular endothelial growth factor in spinal cord injury models in rats. Chin Med J (Engl). 2013; 126(20): 3897-3903, indexed in Pubmed: 24157153.

20. Liu X, Zhou Yi, Wang Z, et al. Effect of VEGF and CX43 on the promotion of neurological recovery by hyperbaric oxygen treatment in spinal cord-injured rats. Spine J. 2014; 14(1): 119-127, doi: 10.1016/j. spinee.2013.06.084, indexed in Pubmed: 24183749.

21. Huang $\mathrm{H}$, Xue L, Zhang $\mathrm{Xu}$, et al. Hyperbaric oxygen therapy provides neuroprotection following spinal cord injury in a rat model. Int J Clin Exp Pathol. 2013; 6(7): 1337-1342, indexed in Pubmed: 23826414.

22. Geng $\mathrm{CK}, \mathrm{Cao} \mathrm{HH}$, Ying $\mathrm{X}$, et al. The effects of hyperbaric oxygen on macrophage polarization after rat spinal cord injury. Brain Res. 2015; 1606: 68-76, doi: 10.1016/j.brainres.2015.01.029, indexed in Pubmed: 25724144.

23. Yang J, Liu X, Zhou Yi, et al. Hyperbaric oxygen alleviates experimental (spinal cord) injury by downregulating HMGB1/NF-KB expression. Spine (Phila Pa 1976). 2013; 38(26): E1641-E1648, doi: 10.1097/ BRS.0000000000000005, indexed in Pubmed: 24335635.

24. Tan J, Zhang F, Liang F, et al. Protective effects of hyperbaric oxygen treatment against spinal cord injury in rats via toll-like receptor 2/ nuclear factor-KB signaling. Int J Clin Exp Pathol. 2014; 7(5): 1911-1919, indexed in Pubmed: 24966901.

25. Wang $Y$, Zhang $S$, Luo $M$, et al. Hyperbaric oxygen therapy improves local microenvironment after spinal cord injury. Neural Regen Res. 2014; 9(24): 2182-2188, doi: 10.4103/1673-5374.147951, indexed in Pubmed: 25657740.

\section{Corresponding author:}

Rita Katznelson, $M D$

Hyperbaric Medicine Unit, Department

of Anaesthesia and Pain Management

Toronto General Hospital

200 Elizabeth Street, EN3-400

M5G 2 C4 Toronto, ON Canada

e-mail: rita.katznelson@uhn.ca

Received: 15.12 .2017

Accepted: 22.03 .2018 


\section{SUPPLEMENTAL RESULTS SECTION}

\section{CASE 1:}

51-year-old male.

Surgical intervention: $3^{\text {rd }}$ time redo left thoracotomy and repair of distal arch and proximal descending thoracic aneurysm with $22 \mathrm{~mm}$ Gelweave graft from left common carotid artery to T7, left subclavian artery bypass with $8 \mathrm{~mm}$ graft. Circulatory arrest time: $21 \mathrm{~min}$. Intraoperative course complicated by bleeding from epidural venous plexus and trivial CSF leak, resolved with gentle packing, Gelfoam (Pharmacia and Upjohn Company, Kalamazoo, Michigan, USA), Surgicel (Ethicon, Sommerville, NJ, USA) and Evicel (Ethicon, Sommerville, NJ, USA).

CSF drainage: Not inserted

Spinal cord deficits: severe weakness in left leg, mild weakness in right leg, bilateral sensory dysfunction in both legs, bowel incontinence, urinary retention.

Onset of spinal cord injury signs/symptoms: observed approximately 12 hours after the end of surgery, on postoperative day (POD) \#1.

Initiation of HBOT treatment: approximately 11 hours after onset of spinal cord injury signs/symptoms.

Number of HBOT treatments: 11, at 2.4 ATA.

Outcome: full motor recovery, partial sensory recovery, intact bowel function, persistent urinary retention requiring intermittent catheterization.

\section{CASE 2}

62-year-old male.

Surgical intervention: Repair of extent II TAAA with 28 $\mathrm{mm}$ Coselli branched Gelweave graft. Reimplantation of celiac, superior mesenteric artery, left and right renal arteries with individual bypass grafts. Bifurcated distal aorto-iliac repair replacing left and right common iliac arteries to their bifurcation. No patent intercostal arteries to re-implant. Use of left heart bypass.

CSF drainage: instituted pre-operatively.

Spinal cord deficits: intraoperative loss of somato-sensory evoked potentials (SSEP) and motor evoked potentials (MEP) to the legs, with recovery of SSEP but not MEP before the end of surgery. On awakening from anaesthesia, after the end of surgery, no movement of left leg, severe weakness of right leg.

Onset of spinal cord injury signs/symptoms: intraoperatively (approximately 8 hours prior to the end of surgery).

Initiation of HBOT treatment: immediately after the end of surgery (direct transfer from the operating room to the HBOT unit).

Number of HBOT treatments: 4, at 2.4 ATA.

Outcome: full motor recovery by POD \#2.

\section{CASE 3}

67-year-old male.

Surgical intervention: Emergent open repair of ruptured abdominal aortic aneurysm, right femoral artery embolectomy

CSF drainage: instituted post-operatively, approximately 11 hours after onset of spinal cord injury signs/symptoms. The patient was deeply sedated for severe hypoxemia and was difficult to assess neurologically. MRI of spinal cord was performed to confirm the diagnosis of spinal cord ischaemia prior to CSF drain insertion.

Spinal cord deficits: paraplegia.

Onset of spinal cord injury signs/symptoms: observed approximately 21 hours after the end of surgery, on postoperative day (POD) \#1.

Initiation of HBOT treatment: on POD \#2, approximately 24 hours after observation of spinal cord injury.

Number of HBOT treatments: 4, at 2.4 ATA.

Outcome: deceased due to multi-organ failure. No evidence of recovery of paraplegia after HBOT treatment.

\section{CASE 4}

53-year-old male.

Surgical intervention: Repair of extent IV TAAA with 26 mm Gelweave graft (VASCUTEK, TERUMO, Inchinnan, Scotland, UK), repair of left common femoral artery. Previous thoracic endovascular aortic repair (TEVAR) from left subclavian to distal descending thoracic aorta for type B aortic dissection. No patent intercostal arteries to re-implant. Celiac, superior mesenteric artery, and right renal arteries re-implanted as a Carrel patch. Left renal artery re-implanted as a button.

CSF drainage: instituted pre-operatively; removed on POD \#5, given lack of symptoms or signs of spinal cord injury; re-instituted on POD \#7, approximately 4 hours after onset of spinal cord injury signs/symptoms.

Spinal cord deficits: left leg weakness.

Onset of spinal cord injury signs/symptoms: observed on POD \#7.

Initiation of HBOT treatment: on POD \#8, approximately 24 hours after onset of spinal cord injury signs/symptoms.

Number of HBOT treatments: $9 ; 2$ treatments at 2.8 ATA, 7 treatments at 2.4 ATA.

Outcome: full recovery of motor function after the last HBOT treatment.

\section{CASE 5}

41-year-old male.

Surgical intervention: Repair of extent II TAAA with 28 $\mathrm{mm}$ Coselli branched Gelweave graft (VASCUTEK, TERUMO, Inchinnan, Scotland, UK) using left heart bypass. T10-T11 
intercostal artery bypass with $10 \mathrm{~mm}$ Dacron graft due to intraoperative loss of MEP and SSEP signals, with full intraoperative recovery of evoked potentials. Aorto-left subclavian artery bypass with $8 \mathrm{~mm}$ graft (near occlusion of previous left subclavian artery graft). Two previous sternotomies: (1) repair ascending aorta for type A dissection; (2) redo sternotomy + valve-sparing root + total arch repair for aneurysmal degeneration.

CSF drainage: instituted pre-operatively.

Spinal cord deficits: paraplegia.

Onset of spinal cord injury signs/symptoms: Observed on POD \#2, approximately 32 hours after the end of surgery.

Initiation of HBOT treatment: POD \#2, approximately 4 hours after the onset of spinal cord injury signs/symptoms. Number of HBOT treatments: $11 ; 5$ treatments at 2.8 ATA, 6 treatments at 2.0 ATA.

Outcome: moderate weakness in hip flexors and knee extensors bilaterally, mild weakness in right ankle dorsiflexors, long toe extensor and ankle plantar extensor. Normal bladder and bowel function. Fully independent on activities of daily living and working full-time.

\section{CASE 6}

72-year-old female.

Surgical intervention: endovascular repair of extent II TAAA with 4-vessel fenestrated graft.

CSF drainage: instituted pre-operatively.

Spinal cord deficits: right leg weakness.

Onset of spinal cord injury signs/symptoms: observed on POD\#1, approximately 24 hours after the end of surgery.
Initiation of HBOT treatment: on POD\#1, approximately 8 hours after onset of spinal cord injury signs/symptoms.

Number of HBOT treatments: 1, at 2.0 ATA.

Outcome: deceased on POD \#2 following cardiac arrest in the setting of massive acute myocardial infarction. Unable to assess spinal cord deficits post HBOT treatments due to clinical deterioration with need for sedation and mechanical ventilation.

\section{CASE 7}

62-year-old male.

Surgical intervention: open repair of extent IVTAAA with previous TEVAR from left subclavian artery to distal descending thoracic aorta, and repair of bilateral common iliac artery aneurysms.

CSF drainage: instituted pre-operatively.

Spinal cord deficits: bilateral severe leg weakness, with altered sensation, bowel and bladder dysfunction.

Onset of spinal cord injury signs/symptoms: POD \#2, approximately 30 hours after the end of surgery.

Initiation of HBOT treatment: POD \#2, approximately 16 hours after onset of spinal cord injury signs/symptoms.

Number of HBOT treatments: 2, at 2.4 ATA.

Outcome: full sensory and motor recovery in right leg with exception of mild weakness on ankle dorsiflexors and long toe extensors; mild weakness in left leg hip flexors, knee extensors, ankle plantar flexors, and moderate weakness in ankle dorsiflexors and long toe extensors; persistent left foot paraesthesia; walking without assistance; indwelling foley catheter for persistent bladder dysfunction; full recovery of bowel function. 